\title{
Hepatic Artery Variations in a Sample of Pakistani Population
}

\author{
Faisal Hanif, Umer Farooq, Awais Amjad Malik, Asghar Khan, Raza Hasnain Sayyed and Imran Khalid Niazi \\ Department of Surgical Oncology and Radiology, Shaukat Khanum Memorial Cancer Hospital \& Research Centre, Lahore, Pakistan
}

\begin{abstract}
Objective: To identify the frequency of different arterial variants of common hepatic artery (CHA) overview identified at abdominal CT angiography (CTA) performed in a large series of patients undergoing CT scans for various reasons.

Study Design: Observational study.

Place and Duration of Study: Department of Surgical Oncology and Radiology, Shaukat Khanum Memorial Cancer Hospital \& Research Centre, Lahore, Pakistan, from October 2016 to September 2018.

Methodology: Findings in randomly selected 1000 patients who underwent CTA were retrospectively evaluated. The pattern of aortic origin of branches of the celiac trunk and superior mesenteric arteries was analysed. The CHA anatomy was then investigated. Hepatic artery anomalies were classified by the Hiatt's method.

Results: There were 629 males and 371 females with a median age of 54 years. Type 1 variation (normal anatomy) was seen in 644 patients. Type 2 variation (left hepatic artery, LHA, aberrations), was seen in 135 patients. LHA was seen arising from left gastric artery or common hepatic artery $(\mathrm{CHA})$ in most of the cases. One patient had a replaced LHA from superior mesenteric artery (SMA). Sixteen patients had an accessory LHA and 119 had a replaced LHA. Type 3 variation (unusual anatomy of right hepatic artery, RHA) was seen in 121 patients, 7 patients had an accessory and 114 had a replaced RHA. The replaced or accessory right hepatic artery originated anywhere from SMA, celiac trunk, aorta, CHA or gastroduodenal artery (GDA). Type 4 variation (unsual origin of both RHA and LHA) was seen in 73 patients. Type 5 variation (CHA arising from SMA) was seen in 23 patients. Type 6 variation (CHA arising directly from aorta) was seen in four patients. Conclusion: Arterial variations are common in our population of patients. A detailed understanding and knowledge of these variations is necessary for surgeons and radiologists to avoid any inadvertent injuries during various procedures.
\end{abstract}

Key Words: Hepatic artery variations, Pakistan, Lower middle income country, Hiatt's classification, South Asian population.

How to cite this article: Hanif F, Farooq U, Malik AA, Khan A, Sayyed RH, Niazi IK. Hepatic artery variations in a sample of Pakistani population. J Coll Physicians Surg Pak 2020; 30(2):187-191.

\section{INTRODUCTION}

Patterns of arterial blood supply to the liver are variable. It is very important to keep the integrity of the hepatic artery blood supply in patients undergoing surgery on the liver or pancreas. Adequate knowledge of hepatic artery anomalies is crucial to avoid injuring them during surgery.

Since, Haller ${ }^{1}$ first time described celiac axis variations, the importance of hepatic artery anomalies has become an area of great interest and research. Michael et al. ${ }^{2}$ also presented a classification for hepatic artery anomalies in 1955 based on dissecting 200 cadavers. ${ }^{2}$ Currently, the most accepted classification of aberrant hepatic arteries is by Hiatt who in 1994 described 6 variations in hepatic artery anatomy. ${ }^{3}$ Song et al. in 2010 published their data on hepatic artery anomalies in over 5000 patients. 4 The accurate understanding of hepatic arterial supply has become crucial part of modern inter-

Correspondence to: Dr. Faisal Hanif, Department of Surgical Oncology, Shaukat Khanum Memorial Cancer Hospital \&

Research Centre, Lahore, Pakistan

E-mail: faisal.hanif@ymail.com

Received: August 29, 2018; Revised: July 31, 2019;

Accepted: August 02, 2019 ventional radiology procedures, liver resection and transplantation. ${ }^{5-8}$

The role of CTA in determining tumor resectability in patients with pancreatic and hepatobiliary malignancy is well established. ${ }^{8}$ CTA also is now routinely used in assessing the variations in hepatic arterial anatomy, which can be found frequently in normal population. Detection of aberrant hepatic artery before interventional procedures or surgery not only helps in surgical planning, decision making regarding choice of procedure, but also prevents potential iatrogenic injuries. ${ }^{9-12}$

The purpose of this study was to determine the frequency of different arterial variants identified at abdominal CT performed in a large series of patients in the local population.

The aim of this study was to determine the variations of extrahepatic arterial anatomy in 1000 Pakistani patients undergoing CTA of the liver for various reasons.

\section{METHODOLOGY}

The study was carried out at the Department of Surgical Oncology and Radiology, Shaukat Khanum Memorial Cancer Hospital and Research Centre, Lahore, Pakistan, from October 2016 to September 2018. Institutional Review 
Board approval was obtained, and the requirement for informed patient consent was waived. Findings in randomly selected 1000 patients who underwent CTA, were retrospectively evaluated. The pattern of aortic origin of branches of the celiac trunk and superior mesenteric arteries was analysed. The CHA anatomy was then investigated. Aberrant arteries were classified further in to: the accessory and the replacing ones. A vessel that supplies a lobe in addition to its normal one was labelled as the accessory hepatic artery, while the replaced hepatic artery was the only branch provides the sole blood supply to that lobe, but originates from other than the normal position. Hepatic artery anomalies were classified by the Hiatt's method. ${ }^{3}$ Descriptive statistics were determined.

\section{RESULTS}

There were 629 males and 371 females with a median age of 54 years. Arterial variations were classified as one of six types (Figure 1). Type 1 variation was seen in 644 patients. In this normal pattern, the common hepatic artery arose from the celiac axis to form the gastroduodenal and proper hepatic arteries; the proper hepatic artery divided distally into right and left branches. Type 2 variation was seen in 135 patients. In this, the left hepatic artery was either replaced or accessory. LHA was seen arising from left gastric artery or CHA (Table la). One patient had a replaced left artery originating from SMA (Figure 2a). Sixteen patients had an accessory left hepatic artery and 119 had a replaced left hepatic artery. Type 3 variation was seen in 121 patients. In this variation,
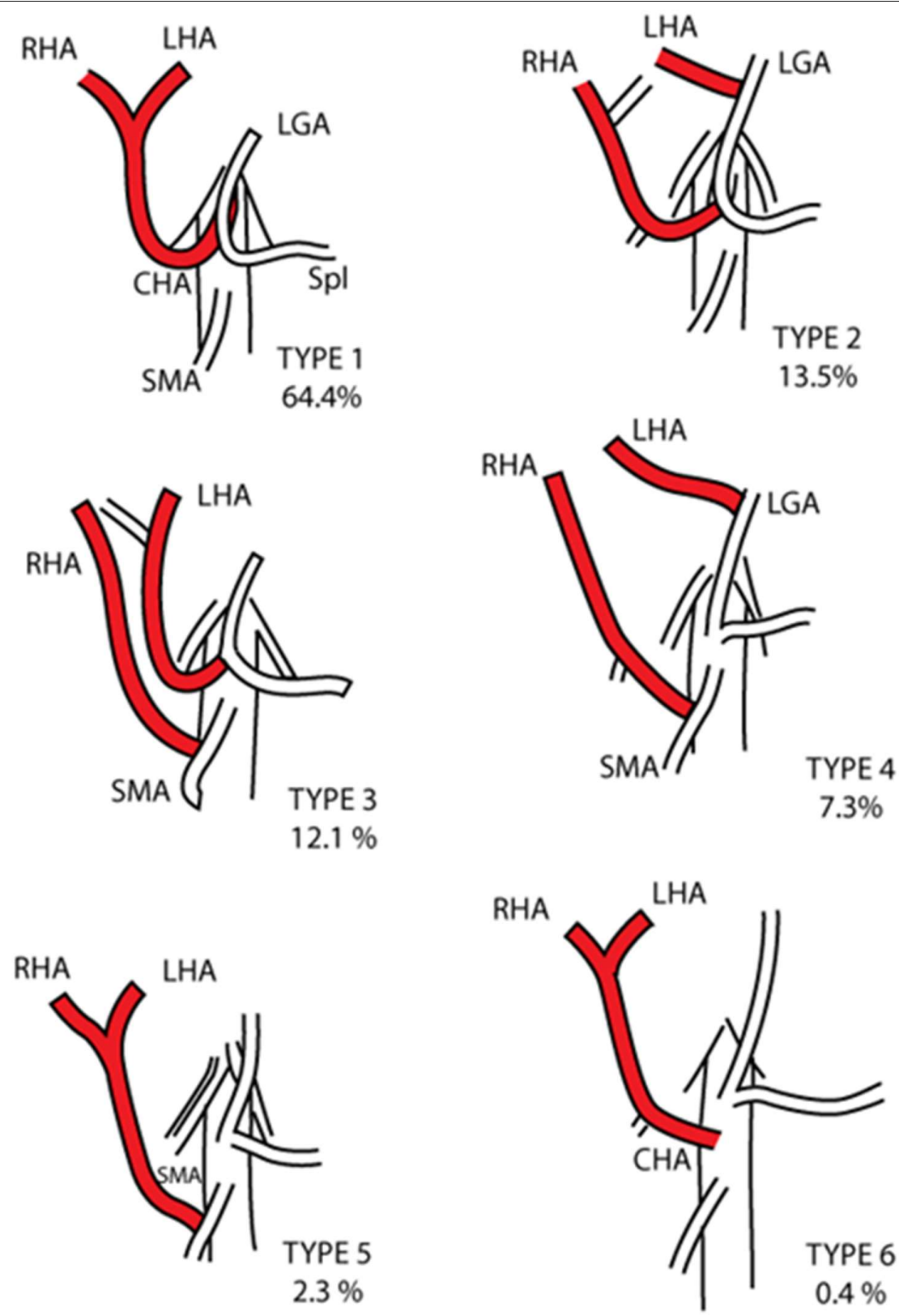

Figure 1: Types of variations in hepatic artery anatomy: Left hepatic artery LHA, Right hepatic artery RHA, Common hepatic artery CHA, Superior mesenteric artery SMA. 
there was a replaced or accessory right hepatic artery. Of these, 7 patients had an accessory and 114 had a replaced right hepatic artery. The variation in origin of right hepatic artery is given in Table la and Figure 2d. Type 4 variation was seen in 73 patients. In this doublereplaced pattern, the right hepatic artery arose from the superior mesenteric artery, and the left hepatic artery was a branch of the left gastric artery (Figure 2e). Type 5 variation was seen in 23 patients. The entire common hepatic artery originated as a branch of the superior

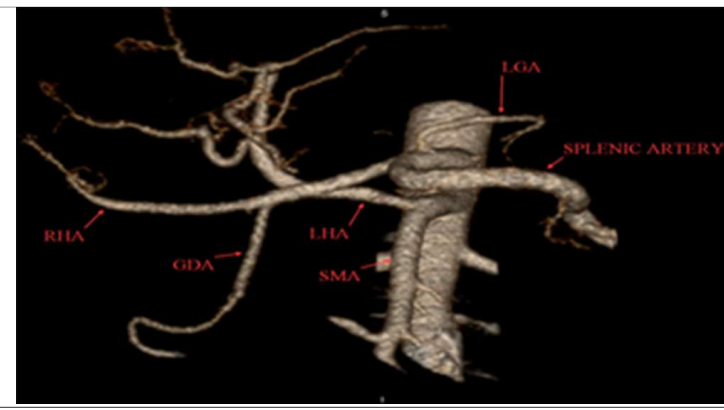

Figure 2a: Reconstructed image from CT of patient who had replaced LHA from SMA.
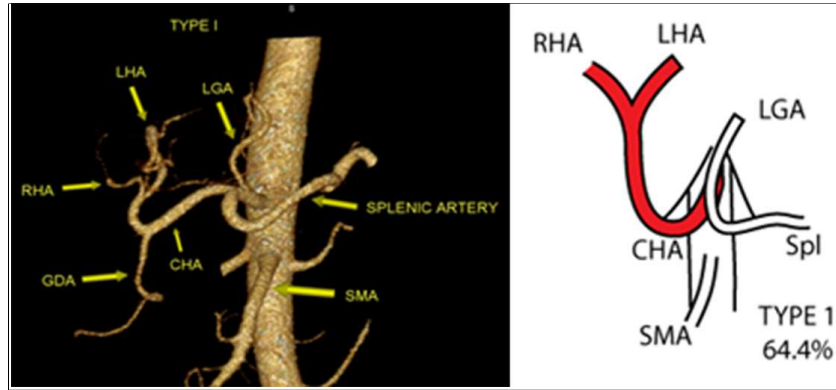

Figure 2b:: Type 1 - Usual hepatic artery anatomy.
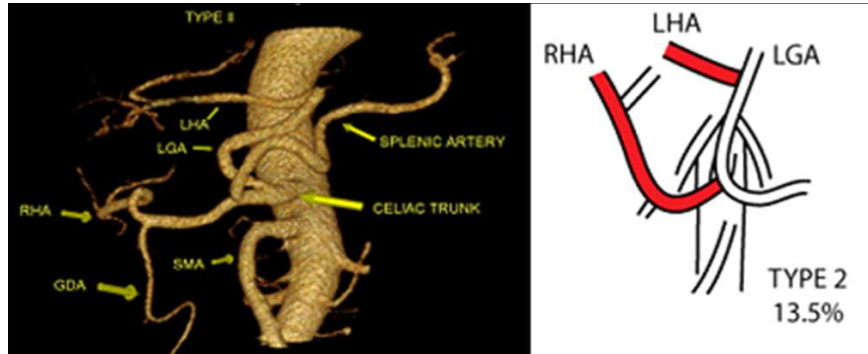

Figure 2c: Type 2 - LHA from LGA.
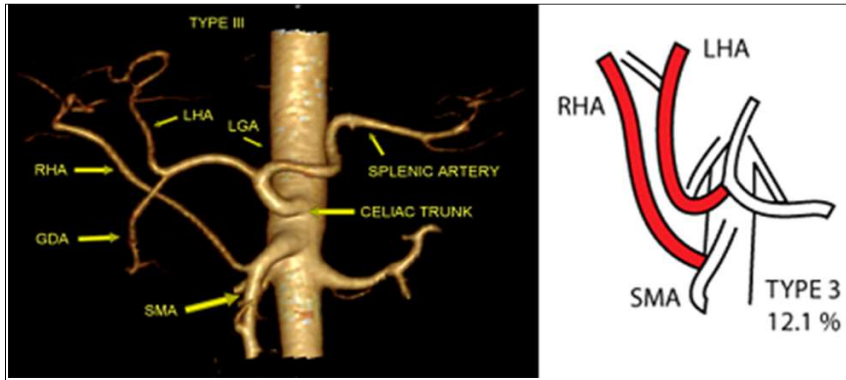

Figure 2d: Type 3 - RHA from SMA. mesenteric artery (Figure 2f). Type 6 variation was seen in four patients. The common hepatic artery took direct origin from the aorta (Figure $2 \mathrm{~g}$ ).
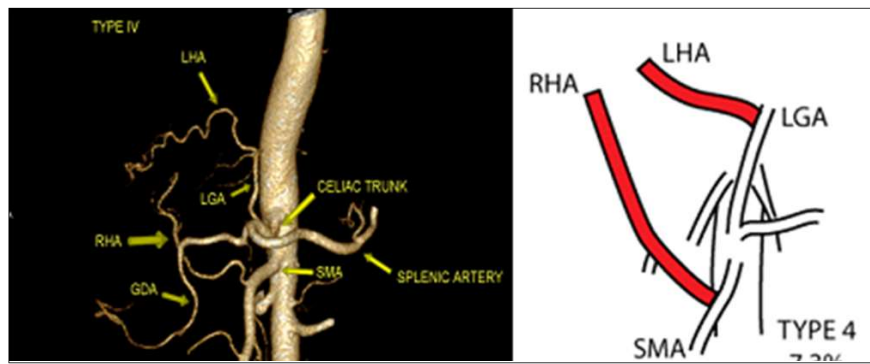

Figure 2e: Type 4 - LHA from LGA + RHA from SMA.
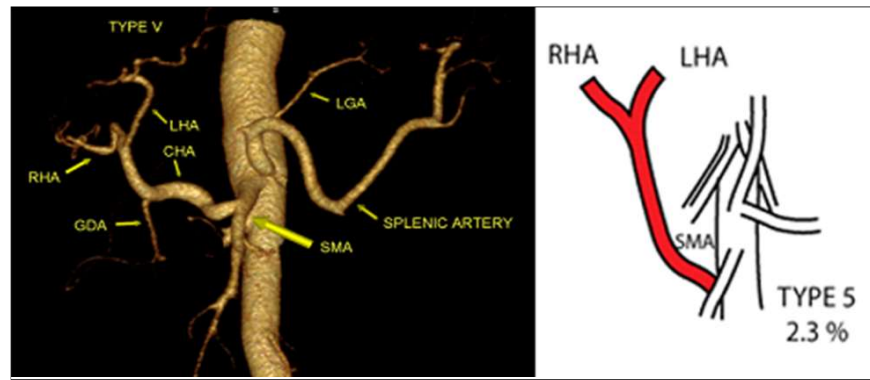

Figure 2f: Type 5 - CHA from SMA.
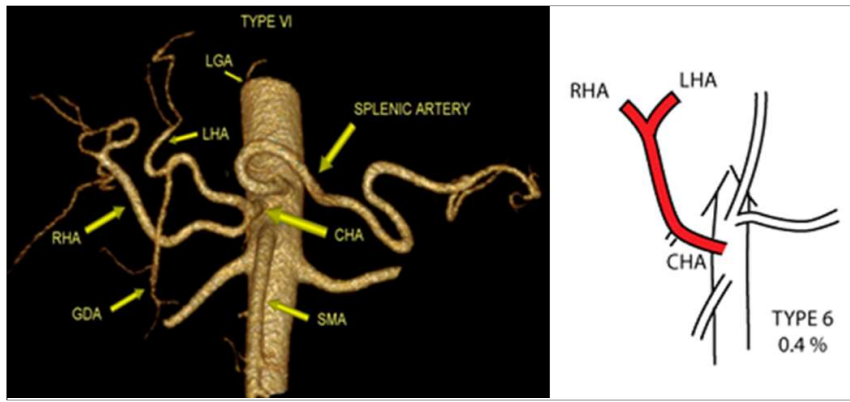

Figure 2g: Type 6 - CHA from Aorta.

Table la: Origin of replaced or accessory LHA (Type 2 variation) and replaced or accessory RHA (Type 3 variation) Right hepatic artery RHA, Common hepatic artery CHA, Gastroduodenal artery GDA.

\begin{tabular}{l|c|l|c}
\hline Type II variation & $\begin{array}{c}\mathrm{N}=135 \\
(\text { Percent) }\end{array}$ & Type III variation & $\begin{array}{c}\mathrm{N}=121 \\
\text { (Percent) }\end{array}$ \\
\hline LHA from left gastric & $133(98.5 \%)$ & RHA from SMA & $109(90 \%)$ \\
LHA from SMA (replaced) & $1(0.7 \%)$ & RHA from celiac trunk & $7(5.7 \%)$ \\
LHA from CHA (accessory) & $1(0.7 \%)$ & RHA from Aorta & $3(2.4 \%)$ \\
& & RHA from CHA (accessory) & $1(0.8 \%)$ \\
& & RHA from GDA & $1(0.8 \%)$ \\
\hline
\end{tabular}

Table lb: A breakdown of left and right hepatic arteries aberrations within Type 2 and 3 category respectively.

\begin{tabular}{l|c}
\hline Aberrations in LHA and RHA & N (Percent) \\
\hline Replaced LHA (Type 2) & $119(88.1 \%)$ \\
Accessory LHA (Type 2) & $16(11.85 \%)$ \\
Replaced RHA (Type 3) & $7(5.7 \%)$ \\
Accessory RHA (Type 3) & $114(94.2 \%)$ \\
\hline
\end{tabular}


Table II: Comparison with Michels's and Hiatt's series.

\begin{tabular}{l|c|c|c}
\hline Type & $\begin{array}{c}\text { Current series } \\
\mathrm{N}=1000(\%)\end{array}$ & $\begin{array}{c}\text { Michels's Series 1962 } \\
\mathrm{N}=200\end{array}$ & $\begin{array}{c}\text { Hiatt's Series } \\
1994 \mathrm{~N}=1000\end{array}$ \\
\hline Type I & $644(64.4 \%)$ & $55 \%$ & $75.7 \%$ \\
Type II & $135(13.5 \%)$ & $18 \%$ & $9.7 \%$ \\
Type III & $121(12.1 \%)$ & $18 \%$ & $10.6 \%$ \\
Type IV & $73(7.3 \%)$ & $04 \%$ & $2.3 \%$ \\
Type V & $23(2.3 \%)$ & $2.5 \%$ & $1.5 \%$ \\
Type VI & $04(0.4 \%)$ & $0.5 \%$ & $0.2 \%$ \\
\hline
\end{tabular}

Sub-classifications of left and right hepatic, arteries, according to their origin and type are described in Table Ib. A side-by-side comparison of the variations seen by Michelle and Hiatt was compared with the variations in Pakistani population in Table II.

\section{DISCUSSION}

Hepatic artery variations have a high incidence. Awareness about these variations is of utmost importance in patients undergoing liver transplantation, laparoscopic surgeries, liver resections, pancreatico-duodenectomy, radiological interventions, and penetrating injuries to the abdomen.

Michele and Hiatt reported incidence of hepatic artery variations as $45 \%$ and $24.3 \%$ patients, respectively. 2,3 In this series, $35.6 \%$ patients had some sort of variation in the origin of their hepatic artery. Acquaintance with aberrant hepatic arterial anatomy is the key to reduce potential hepatic artery injuries.

The most common variation seen in this series was LHA arising from left gastric artery. It was seen as a solitary aberration in 134 patients, and in combination with an aberrant right hepatic arterial anatomy in 73 patients. Michele and Hiatt have classified these variations as Type 2 when encountered alone, and type 4 when encountered in combination with an aberrant right hepatic arterial anatomy.2,3

For RHA, the most common anomaly that has been reported is its origin from the SMA. However, in this series, there were multiple variations in the origin of the $\mathrm{RHA}$. Although the most common variation was its origin from SMA in 109 patients, but this was followed by RHA, originating directly from the celiac trunk in seven patients. In three patients, RHA was directly originating from the aorta. One patient had an accessory RHA, also arising from the $\mathrm{CHA}$. One patient had a $\mathrm{RHA}$ originating inferiorly from GDA.

The above variations have been described before with RHA from SMA being the most common anomaly.4,6,8 The abnormal course of right hepatic artery has significant practical implications for practising surgeons and interventional radiologists. It is crucial to recognise a replaced RHA from SMA during pancreateicoduodenectomy in order to avoid inadvertent injury, ${ }^{13}$ or during resection of liver and performing donor surgery for living donor liver transplant or cadaveric split liver transplant. ${ }^{12}$ Apart from the replaced left or right hepatic arteries, the main hepatic trunk can also be replaced. Twenty-three $(2.3 \%)$ patients has a CHA arising from the SMA and 4 patients had a CHA arising directly from aorta.

Knowledge of all these anomalies is of utmost importance not only for the surgeons but also the radiologists. It is important to prevent injury to the hepatic vascular anatomy while harvesting and re-anastomosing orthotopic liver transplants; avoiding vascular injury in laparoscopic cholecystectomies and preventing complications during vascular radiological procedures. $5,7,11,14$

CTA has an important role in delineating these vascular anomalies before undertaking any sort of surgical or radiological procedures. CT angiography has a reported accuracy of $97-98 \%$ compared with conventional angiography for detecting arterial variants.6,8,9 In addition to evaluating for vascular involvement by tumor, it also assesses for the presence of variant arterial anatomy. Patient management can be completely altered due to the presence of aberrant arterial anatomy. Therefore, a detailed study of the preoperative CTA of the patient may give detailed awareness of these variations. This knowledge may obviate extensive dissection to identify the vessels and avoid any vascular disaster. ${ }^{15}$

\section{CONCLUSION}

Presence of hepatic artery variations in $35.6 \%$ of our population patients requires that all surgeons performing hepatopancreaticobiliary and transplant surgeries, and radiologists skilled in interventions, should be well aware of all these variations beforehand. Detailed knowledge of these hepatic arterial patterns may help avoid unnecessary complications, which in turn will help achieve better surgical and radiological outcomes.

\section{ETHICAL APPROVAL:}

The paper is compliant with ethical framework and guidelines of the institution in relation with publication of medical research.

\section{CONFLICT OF INTEREST:}

Authors declared no conflict of interest.

\section{AUTHORS' CONTRIBUTION:}

FH: Contributed in study design, data analysis and manuscript writing.

UF: AAM, RHS: Collected data and performed analysis. IKN, AK: Reviewed scans.

\section{REFERENCES}

1. Chen H, Yano R, Emura S, Shoumura S. Anatomic variation of the celiac trunk with special reference to hepatic artery patterns. Ann Anat 2009; 191:399-407. 
2. Michels NA. Newer anatomy of the liver and its variant blood supply and collateral circulation. Am J Surg 1962; 112: 337-47.

3. Hiatt JR, Gabbay J, Busuttil RW. Surgical anatomy of the hepatic arteries in 1000 cases. Ann Surg 1994; 220:50-52.

4. Song SY, Chung JW, Yin YH, Jae HJ, Kim HC, Jeon UB, et al. Celiac axis and common hepatic artery variations in 5002 patients: Systematic analysis with spiral CT and DSA. Radiology 2010; 255:278-88.

5. Lee KH. Transcatheter arterial chemoembolization for hepatocellular carcinoma: Anatomic and hemodynamic considerations in the hepatic artery and portal vein. Radiographics 2002; 22: 1077-91.

6. Winston CB, Lee NA, Jarnagin WR, Teitcher J, DeMatteo RP, Fong $\mathrm{Y}$, et al. CT angiography for delineation of celiac and superior mesenteric artery variants in patients undergoing hepatobiliary and pancreatic surgery. AJR Am J Roentgenol 2007; 189:W13-9.

7. Huang $Y$, Liu C, Lin J. Clinical significance of hepatic artery variations originating from the superior mesenteric artery in abdominal tumor surgery. Chin Med J 2013; 126:899-902.

8. DeCecco CN. Anatomic variations of the hepatic arteries in 250 patients studied with 64-row CT angiography. Eur Radiol 2009; 19:2765-70.
9. Raptopoulos V, Steer ML, Sheiman RG, Vrachliotis TG, Gougoutas CA, Movson JS. The use of helical CT and CT angiography to predict vascular involvement from pancreatic cancer: Correlation with findings at surgery. AJR 1997; 168:971-7.

10. Zhou L. Enhanced therapeutic efficacy of combined use of sorafenib and transcatheter arterial chemoembolization for treatment of advanced hepatocellular carcinoma. Jpn J Clin Oncol 2014; 44:711-7.

11. Yang F, Long J, Fu DL, Jin C, Yu XJ, Xu J, et al. Aberrant hepatic artery in patients undergoing pancreaticoduo-denectomy. Pancreatology 2008; 8:50-4.

12. Varotti G, Gondolesi GE, Munoz L, Florman S, Fishbein TM, Emre $S$, et al. Biliary complications in 96 right lobe living donor liver transplants. J Gastrointest Surg 2003; 7:271.

13. Moon JJ, Widjicks CA, Williams JM. Right hepatic artery branching off the superior mesenteric artery and its potential implications. IJAV 2009; 2:143-5.

14. Shukla PJ, Barreto SG, Kulkarni A. Vascular anomalies encountered during pancreatoduodenectomy: Do they influence outcomes? Ann Surg Oncol 2010; 17:186-93.

15. Winston B, Lee CA, Jarnagin NR, Teitcher W, DeMatteo JP, Fong $\mathrm{R}$, et al. CT angiography for delineation of celiac and superior mesenteric artery variants in patients undergoing hepatobiliary and pancreatic surgery. Am J Roentgenol 2007; 189:W13-9. 